\title{
METODOLOGIA PARA MAPEAMENTO DE VULNERABILIDADE COSTEIRA À ELEVAÇÃO DO NÍVEL MÉDIO DO MAR (NMM) EM ESCALA LOCAL
}

\section{Methodology for mapping the coastal vulnerability due to mean sea level rise on local scale}

\author{
Marcelo Soares Teles Santos ${ }^{1}$ \\ Venerando Eustáquio Amaro ${ }^{2}$ \\ Anderson Targino da Silva Ferreira ${ }^{2}$ \\ Andressa de Abreu Barboza ${ }^{3}$ \\ Marília Crusoé Figueiredo ${ }^{3}$ \\ Alana Grochowalski Araújo ${ }^{3}$ \\ ${ }^{1}$ Universidade Federal do Sul da Bahia (UFSB) Centro de Formação em Tecnociências e Inovação \\ (CFTI) Câmpus Universitário, CEP 45613-204, Itabuna /BA Email: marcelostsantos@gmail.com
${ }^{2}$ Universidade Federal do Rio Grande do Norte - UFRN Departamento de Geologia (DGEO) Campus Universitário, CEP 59078-970, Natal/RN
${ }^{3}$ Universidade Federal do Recôncavo da Bahia (UFRB) Centro de Ciências Exatas e Tecnológicas \\ (CETEC) Câmpus Universitário, CEP 44380-000, Cruz das Almas/BA
}

marcelostsantos@gmail.com

\section{Resumo:}

Este trabalho apresenta metodologia para mapeamento da vulnerabilidade costeira à elevação do Nível Médio do Mar (NMM) em escala local, com estudo de caso na orla marítima da Ilha de Madre de Deus/BA. Essa orla marítima é densamente ocupada por bairros residenciais e instalações da indústria petrolífera. Para isso, foi criado um índice de vulnerabilidade costeira com a combinação de quatro variáveis geológicas (geomorfologia, declividade, elevação e taxa de erosão/acreção na face de praia), determinadas em levantamentos sazonais (verão e inverno) de perfis de praia georreferenciados e niveladas em relação ao NMM, e três variáveis de processos físicos costeiros (taxa de elevação do nível médio do mar, altura significativa de onda e amplitude de maré média). O mapa representou a variação da vulnerabilidade relativa ao longo de distintos setores da linha de costa possibilitando identificar a combinação mais crítica das variáveis, ou seja, áreas prioritárias às intervenções antrópicas para redução das vulnerabilidades por meio de estratégias de prevenção, mitigação e/ou adaptação socioeconômicas e ambientais aos impactos da elevação do NMM. Os resultados mostraram alto potencial na aplicação da metodologia em setores de beira-mar, cuja pequena extensão requer o mapeamento em escala de detalhe. 
Palavras chaves: Índice de vulnerabilidade costeira, perfil de praia, elevação do nível médio do mar.

\begin{abstract}
:
This paper presents methodology to coastal vulnerability mapping to Mean Sea Level (MSL) rise in local scale, with case study at waterfront of Madre de Deus Island, Baía de Todos os Santos, Bahia, Brazil. This waterfront is densely occupied by residential quarters and oil industry facilities. For this, coastal vulnerability indexes were used based on combination of four geological variables (geomorphology, slope, elevation and erosion/accretion rate at beach face) obtained in seasonal surveys (summer and winter) of georeferenced and levelling beach profiles relative to MSL, and three variables of coastal physical processes (MSL rate of rise, significant wave height and mean average tidal range). The map represented the variation of relative vulnerability over distinct sectors along the coastline making it possible to identify the most critical combination of variables, i.e., i.e., anthropogenic interventions priority areas for reduction of vulnerabilities, through strategies for prevention, mitigation and/or socioeconomic and environmental adaptation to the impacts of MSL rise. The results showed high potential for application of the methodology in waterfront sectors whose small extension requires detail scale mapping.
\end{abstract}

Keywords: Coastal Vulnerability Index, beach profile, mean sea level rise.

\title{
1. Introdução
}

O Quinto Relatório de Avaliação (AR5) do Painel Intergovernamental sobre Mudanças Climáticas (IPCC, na sigla em inglês) advertiu que a superfície da Terra poderia aquecer entre $2,6^{\circ} \mathrm{C}$ e $4,8^{\circ} \mathrm{C}$ ao longo deste século, o que faria com que o nível dos oceanos se elevasse em média entre 52 e 82 centímetros. Estudos anteriores mostraram que o nível médio dos oceanos já subiu em média 20 centímetros entre 1900 e 2012 (IPCC, 2013). Em tal situação, segundo os cenários tratados no AR5 do IPCC, se a taxa de elevação dos oceanos exceder outros 60 centímetros, com a atuação das forçantes hidrodinâmicas, sobretudo os regimes de marés e clima de ondas, o resultado será uma forte erosão nas áreas costeiras de todo o planeta (IPCC, 2013).

Nesse cenário de elevação futura dos oceanos a avaliação da vulnerabilidade à elevação do Nível Médio do Mar (NMM) é ferramenta fundamental para identificar áreas mais susceptíveis a desastres naturais e antecipar impactos socioeconômicos na orla marítima, onde estão instaladas importantes cidades e atividades antrópicas. Apesar disso, a maioria dos municípios costeiros ainda não possuem ferramentas de gestão de desastres naturais, sobretudo da erosão costeira e dos prejuízos socioeconômico-ambientais decorrentes. Nesse sentido, a elaboração do mapa de vulnerabilidade tem grande aplicabilidade por ser capaz de identificar áreas prioritárias para intervenções antrópicas de prevenção e/ou mitigação de impactos provocados por desastres naturais.

Mapeamentos de vulnerabilidades à elevação do NMM em escala regional já foram realizados em várias regiões do planeta como ferramenta de gestão costeira de áreas susceptíveis a desastres naturais. Cita-se, como exemplos, os trabalhos de Boori e Amaro (2011a, 2011b), Boruff et al. (2005), Gornitz et al. (1994), Gorokhovich; Leiserowitz; Dugan (2014), Hammar-Klose et al. 
(2003), Kumar et al. (2010), Pendleton et al. (2010), Shaw et al. (1998), e Thieler e HammarKlose (1999). No entanto, em áreas com dimensões relativamente pequenas, como o caso de municípios de pequeno porte, a metodologia adotada deve permitir o mapeamento da vulnerabilidade na escala local com alto nível de detalhe.

Portanto, este trabalho tem como objetivo apresentar metodologia para mapeamento da vulnerabilidade costeira à elevação do NMM em escala local. Foi utilizado um índice de vulnerabilidade costeira baseado na combinação de variáveis geológicas, determinadas a partir de perfis topográficos georreferenciados e nivelados ao nível médio do mar do Sistema Geodésico Brasileiro (SGB, datum vertical de Imbituba/SC), e três variáveis de processos físicos costeiros, obtidas a partir de dados de agências governamentais e de trabalhos prévios (DHN, 2013; IPCC, 2013; Barboza, 2014).

A aplicação do método foi feita na faixa litorânea da Ilha de Madre de Deus/BA, localizada na porção Norte da Baía de Todos os Santos/BA (Figura 1). A ilha enfrenta forte pressão antrópica nas proximidades da zona de praia provocada pela presença de densos conjuntos residenciais e da intensa atividade petrolífera, com tanques de armazenamento e dutos de transporte de petróleo e derivados, além de um terminal marítimo (Bradley et al., 2000; Freire, 2012). As Figuras 2a e $2 \mathrm{~b}$ ilustram exemplos de instalações antrópicas localizadas nas proximidades da orla costeira. A erosão costeira ocorrida nos últimos anos é tão intensa que, na maré em preamar, o nível d'água já atinge o muro de contenção que protege a orla marítima (Figuras 2a e 2b). Essas características fazem com que a área de estudo seja cada vez mais vulnerável ao processo erosivo provocado pela elevação do nível médio do mar esperado para as próximas décadas.

Em relação aos citados trabalhos anteriores sobre vulnerabilidade à elevação do NMM, a presente metodologia destaca-se por realçar níveis de intensidade de vulnerabilidade relativa em áreas de dimensões relativamente pequenas, na escala local e ao nível de detalhe. Tem ampla aplicabilidade no gerenciamento costeiro de municípios de pequeno porte e/ou com poucos recursos econômicos, que não possuem instalação de sistemas de monitoramento e alerta fundamentais na prevenção de desastres naturais. Segundo Shidawara (1999) e Kobiyama et al. (2006), uma das maiores deficiências relacionadas aos desastres naturais no Brasil é a ausência desses sistemas. 


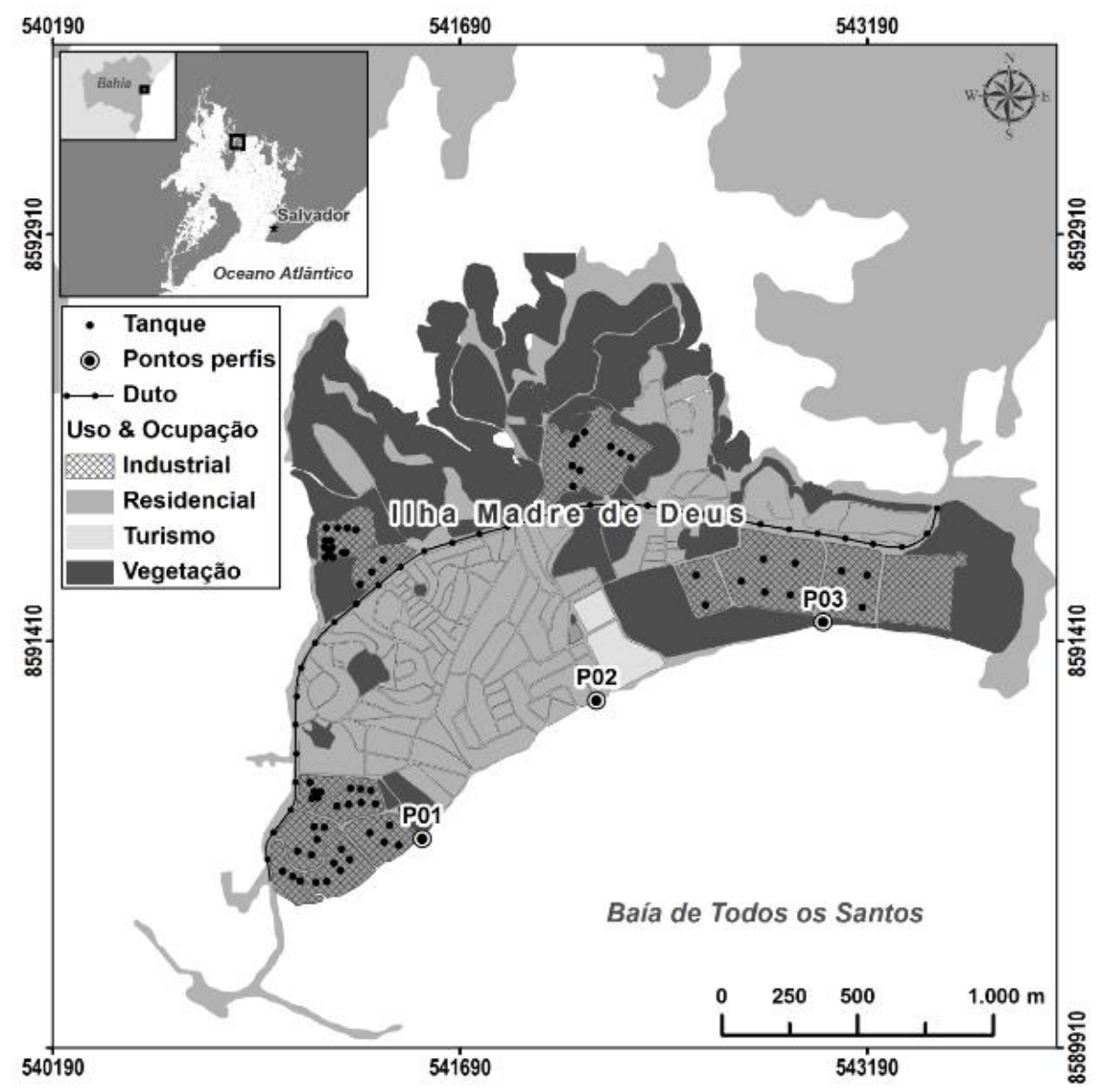

Figura 1: Mapa de Localização da Ilha de Madre de Deus, Baía de Todos os Santos, com localização de alguns pontos da zona de praia sob pressão antrópica (P1, P2 e P3). O ponto P1 é limite entre os setores industrial e residencial, o ponto P2 é limite entre os setores residencial e turísico, e o ponto P3 é setor industrial.
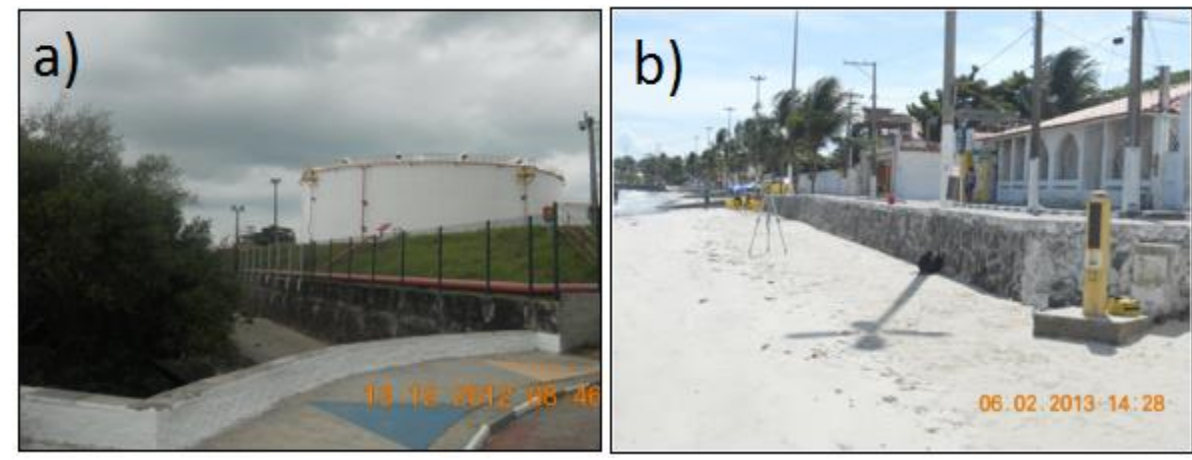

Figura 2: Ilustração de alguns trechos da zona de praia sob pressão antrópica (Pontos P1 e P2 da Figura 1). Em (a) é ilustrado o ponto P1, limite entre os setores industrial e residencial; em (b) o ponto P2, limite entre os setores residencial e turísico. O ponto P3 da Figura 1 é setor industrial com instalações semelhantes às contidas no ponto P1. 


\section{Caraterização da área de estudo}

O Município de Madre de Deus está localizado na porção norte da Baía de Todos os Santos (BTS) e faz parte da Região Metropolitana de Salvador (RMS), além de integrar a Área de Proteção Ambiental Baía de Todos os Santos (APA-BTS). O território do município é composto por três ilhas: Madre de Deus, Maria Guarda e das Vacas, sendo as duas primeiras habitadas, além do Atol do Capeta, que juntas ocupam uma área aproximada de $11,14 \mathrm{~km}^{2}$. A primeira é sede do município e compreende uma área de $3,23 \mathrm{~km}^{2}$, com 17.376 habitantes e densidade populacional de $1.559 \mathrm{hab} / \mathrm{km}^{2}$ (Freire, 2012).

Como apresentado no capítulo introdutório, a zona de praia da Ilha de Madre de Deus sofre forte pressão antrópica devido à presença de densos conjuntos habitacionais e da indústria petrolífera. Essa pressão foi intensificada a partir da década de 60 com a implantação do Terminal Marítimo da PETROBRAS (TEMADRE), instalado na ilha devido a sua proximidade da Refinaria Landulfo Alves, município de São Francisco do Conde. Passou então a ser utilizada para armazenamento, carga e descarrega de navios e caminhões transportadores de petróleo e derivados, tendo a presença marcante de instalações industriais de grande porte no perímetro urbano, como tanques para estocagem e armazenamento, assim como dutovia para transporte de petróleo e derivados originários da citada refinaria (Bradley et al., 2000; Freire, 2012).

Os dados climatológicos e oceanográficos foram obtidos em Hatje e Andrade (2009). De acordo com os dados da estação meteorológica de Ondina (Salvador/BA), o clima na entrada da BTS, no canal de Salvador, é tropical-úmido, com médias anuais de temperatura, precipitação e evaporação de $25,2^{\circ} \mathrm{C}, 2.100 \mathrm{~mm}$ e $1.002 \mathrm{~mm}$, respectivamente. As temperaturas atingem valores máximos nos meses de janeiro, fevereiro e março (cerca de $30^{\circ} \mathrm{C}$ ), e valores mínimos nos meses de julho, agosto e setembro (em torno de $21^{\circ} \mathrm{C}$ ). As menores precipitações $(<150 \mathrm{~mm}$ ) ocorrem em janeiro e setembro e as maiores em abril, maio e junho (>300 mm), quando ocorre cerca de $40 \%$ da precipitação média anual. A série histórica de intensidade e direção de ventos (média mensal) da estação de Ondina (INMET, 2015), desde 31 de agosto de 1963 até 31 de janeiro de 2015, indicaram uma direção de $14^{\circ}(\mathrm{NNE})$ variando entre $0^{\circ}(\mathrm{N})$ a $32^{\circ}(\mathrm{NE})$, com velocidade média de $2 \mathrm{~m} / \mathrm{s}$, mínima de $1 \mathrm{~m} / \mathrm{s}$ e máxima de $15 \mathrm{~m} / \mathrm{s}$.

\section{Metodologia experimental}

A vulnerabilidade pode ser definida como a susceptibilidade ou a pré-disposição intrínseca de um elemento ou sistema exposto a um perigo ser afetado gravemente (KUMAR et al., 2010). Para quantificar a vulnerabilidade costeira às mudanças físicas (erosão e/ou acreção) devido à futura elevação do NMM foi adotado o índice de vulnerabilidade costeira CVI (da sigla em inglês Coastal Vulnerability Index), similar aos índices usados em Boori e Amaro (2011a), Boruff et al. (2005), Gornitz et al. (1994), Gorokhovich; Leiserowitz; Dugan (2014), HammarKlose et al. (2003), Kumar et al. (2010), Pendleton et al. (2010), Shaw et al. (1998), e Thieler \& Hammar-Klose (1999), que utiliza quatro variáveis geológicas e três variáveis de processos físicos costeiros na seguinte equação: 


$$
C V I=\sqrt{\frac{a^{*} b^{*} c^{*} d^{*} e^{*} f^{*} g}{7}}
$$

onde, $a$ (geomorfologia), $b$ (declividade da praia), $c$ (elevação da praia) e $d$ (taxa de erosão/acreção da zona de praia) são as variáveis geológicas, e $e$ (taxa de elevação relativa do nível do mar), $f$ (altura significativa de onda), e $g$ (amplitude de maré média) são as variáveis de processos físicos costeiros.

Os índices CVI estabelecidos para a área de estudo, com base na Equação 1, foram calculados para cada setor da zona de praia, isoladamente. No cálculo, para cada variável (de $a$ até $g$ ) foi atribuído um valor representativo do nível de vulnerabilidade: valor 3 é alto, 2 é médio e 1 é baixo. Assim, os intervalos de CVI de cada nível de vulnerabilidade (alto, médio e baixo) foram definidos de acordo com os $25^{\circ}, 50^{\circ}$, e $75^{\circ}$ percentil do conjunto de CVI calculados nos diversos setores. Dessa forma, setores com valores de CVI abaixo de $25^{\circ}$ foram atribuídos à categoria de baixa vulnerabilidade, entre $25^{\circ}$ e $75^{\circ}$ de média vulnerabilidade, e maiores do que $75^{\circ}$ de alta vulnerabilidade.

A variável geomorfologia, não numérica, foi determinada qualitativamente pela resiliência da praia em relação ao processo erosivo, avaliada em função da presença de feições responsáveis pelo aprisionamento e/ou reposição de sedimentos ao ambiente, como dunas frontais e bermas, que auxiliam na manutenção do ambiente praial. As variáveis geológicas numéricas (declividade, elevação e taxa de erosão/acreção da zona de praia) foram avaliadas através de perfis topográficos de praia dispostos perpendicularmente à linha de costa e distribuídos ao longo da costa. As informações das variáveis de processos físicos costeiros (taxa de elevação relativa do nível do mar, altura significativa de onda, e amplitude de maré média), que contribuem para alterações na vulnerabilidade à inundação de uma seção particular da linha de costa, foram obtidas nas agências governamentais especializadas e em estudos prévios (DHN, 2013; IPCC, 2013; Barboza, 2014).

Os perfis topográficos foram levantados nos períodos de verão e inverno, estações representativas dos diferentes cenários de atuação dos agentes dinâmicos costeiros (ventos, ondas e correntes de maré e litorâneas) em relação à maior erosão e/ou acreção de sedimentos no ambiente praial, acompanhadas de modificações morfológicas no perfil da praia (Amaro; Santos; Souto, 2012; Santos e Amaro, 2013). Os levantamentos foram executados pelo nivelamento geométrico de precisão com nível de luneta automático (Espartel, 1983; Loch e Cordini, 2007). Para garantir confiabilidade aos resultados, os pontos topográficos dos perfis foram determinados em três sessões de observações, sendo adotada a média aritmética como valor representativo e desvio padrão de $1 \mathrm{~cm}$ como tolerância. Os perfis altimétricos foram georreferenciados e nivelados em relação aos referenciais geodésicos (planimétrico e altimétrico) adotados no Sistema Geodésico Brasileiro (SGB) a partir de pontos de controle, materializados com piquetes e implantados nas proximidades do início de cada perfil. Nesses pontos foram determinadas as coordenadas geodésicas e as altitudes ortométricas de precisão com o emprego do Global Navigation Satellite System (GNSS) e do mapa de alturas geoidais, como se explica a seguir.

As coordenadas geodésicas (latitude, longitude e altitude geométrica) dos pontos de controle foram determinadas pelo posicionamento GNSS relativo estático, no qual dois receptores coletam dados, simultaneamente, durante um determinado intervalo de tempo, sendo um instalado em uma estação de referência (estação base, de coordenadas conhecidas) e o outro no ponto de interesse (estação móvel), conforme Monico (2007), Santos; Amaro; Souto (2011) e Seeber 
(1993). Como referências aos posicionamentos dos pontos de controle foram utilizadas estações geodésicas ativas da Rede Brasileira de Monitoramento Contínuo (RBMC) do SGB. Os receptores utilizados rastrearam observações da fase da onda portadora nas frequências L1 e L2, sessões de 4 horas e uso de efemérides precisas, o que resultou em coordenadas com precisão de poucos centímetros em relação às estações do SGB.

As altitudes geométricas (relacionadas ao elipsóide de referência) dos pontos de controle obtidas pelo GNSS foram convertidas nas altitudes ortométricas (relacionadas ao nível médio do mar) através da altimetria GNSS. O método consiste em determinar a altitude ortométrica $\left(H_{i}\right)$ de um ponto " $i$ " de interesse pela combinação da altitude geométrica $\left(h_{i}\right)$, obtidas por GNSS, e da altura geoidal $\left(N_{i}\right)$, fornecida por um modelo geoidal (Featherstone et al., 1998; Santos et al., 2013):

$$
H_{i}=h_{i}-N_{i}
$$

As alturas geoidais utilizadas foram obtidas com o modelo geoidal do SGB, MAPGEO2010 (IBGE, 2013), e o cálculo das altitudes foi realizado no aplicativo desenvolvido por Santos e Amaro (2011). Avaliações realizadas nas proximidades da área de estudo (Barboza, 2014) e em outras regiões do Nordeste (Santos et al., 2013) indicam que o erro estimado nas alturas geoidais está em torno de alguns decímetros. No entanto, esse erro é praticamente constante na área de estudo devido às dimensões reduzidas (aproximadamente $3 \mathrm{~km}$ ), ou seja, não ocorrem erros relativos nas alturas geoidais ao longo da zona de praia. Nesse caso, de acordo com a Equação (2), os erros relativos da altitude ortométrica (H) são da mesma ordem de grandeza dos erros da altimetria GNSS (h); ou seja, de poucos centímetros. Essa característica da metodologia é essencial para se obter diferenças de nível com acurácia de alguns centímetros ao longo de cada perfil de praia e entre os perfis de praia realizados ao longo da costa

\section{Resultados e Discussões}

A Figura 3 apresenta o mapa de vulnerabilidade costeira à elevação do NMM da Ilha de Madre de Deus, calculada pela Equação (1), no qual a zona de praia foi subdividida em três setores, Leste (S1), Central (S2) e Oeste (S3), em função das semelhanças nas características morfológicas identificadas em imagens de satélites, perfis topográficos perpendiculares à linha de costa e observações visuais. A Tabela 1 apresenta as categorias de vulnerabilidades relativas determinadas para cada variável utilizada na Equação (1) e apresentadas na Figura 3. O uso do solo apresentado na Figura 3 mostra que o setor S1 possui predominantemente instalações industriais, o setor S2 ocupação residencial e o setor S3 a combinação de atividades turísticas, ambientais e industriais. 


\subsection{Vulnerabilidade costeira à elevação do NMM}

Na Figura 3 e na Tabela 1 se observa que apenas as variáveis geológicas quantitativas apresentaram variação relativa ao longo da costa, da seguinte forma: i) a declividade de praia variou entre $1,0 \%$ e $2,3 \%$ no sentido do setor $\mathrm{S} 1$ ao $\mathrm{S} 3$, sendo que áreas com menor declividade (S1) possuem maior vulnerabilidade, pois tendem ao recuo mais acelerado da linha de costa diante da elevação relativa do NMM; ii) a elevação da praia variou entre $0,67 \mathrm{~m}$ e $1,41 \mathrm{~m}$ no sentido do setor S1 ao S3, sendo que áreas com menor elevação de praia (S1) possuem maior vulnerabilidade ao recuo da linha de costa; iii) a taxa de erosão/acreção foi de $0,65 \mathrm{~mm} /$ ano no setor $\mathrm{S} 1,0,12 \mathrm{~mm} /$ ano no setor $\mathrm{S} 2$, e $-0,54 \mathrm{~mm} /$ ano no setor $\mathrm{S} 3$, sendo que áreas com maior taxa de variação da linha de costa possuem maior vulnerabilidade.

A variável geológica geomorfologia (qualitativa) e as variáveis de processos físicos costeiros presentes na Equação 1 não foram apresentadas no mapa de vulnerabilidade da Figura 3 devido ao fato de não terem ocorridas variações ao longo dos três setores da costa. A variável geomorfologia não variou pela ausência de feições morfológicas naturais de proteção costeira, como dunas frontais, bermas e rochas, e ainda a ausência de feições de pós-praia, pois as marés em preamar atingem os muros de arrimo da orla marítima (Figuras 2a e 2b). As variáveis de processos físicos costeiros não se alteraram por causa das dimensões relativamente pequenas da área de estudo e dos padrões uniformes da linha de costa e das variáveis físicas. Na variável taxa de elevação relativa do nível do mar, o IPCC (IPCC, 2013) estima que o aumento do nível no ano 2100 estará entre 0,52 a 0,98m na área de estudo. A variável amplitude média de maré foi de 2,5 m, obtida pela Diretoria de Hidrografia e Navegação (DHN, 2013). Na variável altura significativa de onda os valores medidos em Barboza (2014) indicaram variações de apenas alguns decímetros, pelo fato da área de estudo estar protegida da ação direta da dinâmica de mar aberto (interior de uma baía). 


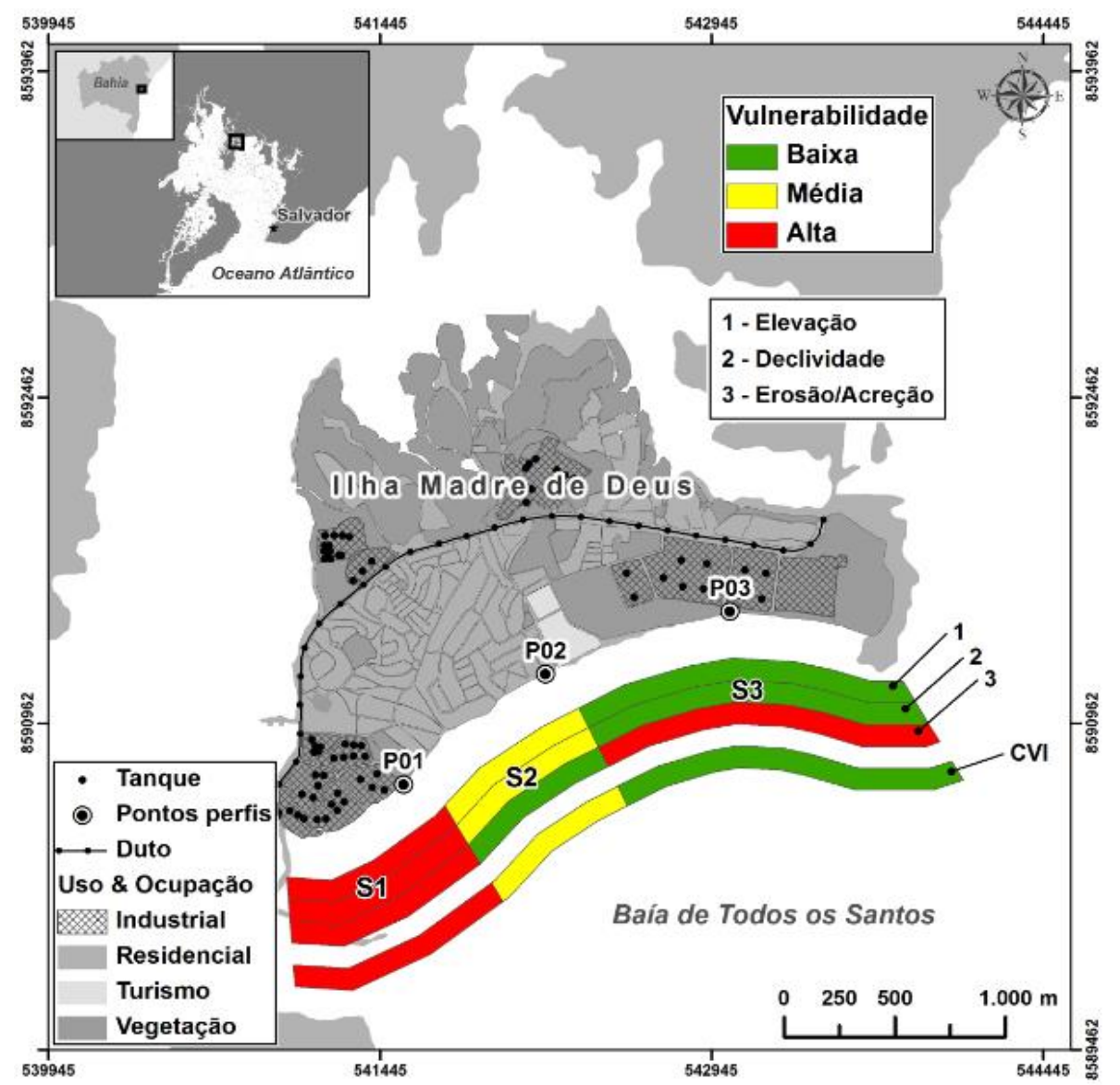

Figura 3: Mapa de vulnerabilidade costeira (3 variáveis e CVI) à elevação do NMM da zona de praia da Ilha de Madre de Deus/BA em função dos setores S1, S2 e S3. Nos pontos P01, P02 e P03 estão localizados os perfis de praia (1, 2 e 3, respectivamente) levantados para cálculo das variáveis geológicas.

Tabela 1: Intervalos das variáveis envolvidas no índice de vulnerabilidade costeira.

\begin{tabular}{l|c|c|c}
\hline \multicolumn{1}{c|}{ Variáveis } & Baixo (1) & Médio (2) & Alto (3) \\
\hline 1 - Geomorfologia & $\mathrm{SC}$ & $\mathrm{SC}$ & $\mathrm{SC}$ \\
\hline 2 - Declividade da praia & $1,8 \%<\mathrm{d}<2,3 \%$ & $1,4 \%<\mathrm{d}<1,8 \%$ & $1,0<\mathrm{d}<1,4 \%$ \\
\hline 3 - Elevação da praia & $1,41<\mathrm{h}>1,16 \mathrm{~m}$ & $0,91 \mathrm{~m}<\mathrm{h}<1,16 \mathrm{~m}$ & $0,67<\mathrm{h}<0,91 \mathrm{~m}$ \\
\hline 4 - Erosão/Acreção & $0,18<\mathrm{t}>0,30$ & $0,30<\mathrm{t}>0,47$ & $0,47<\mathrm{t}>0,65$ \\
\hline 5 - Elevação relativa do NMM & $\mathrm{SC}$ & $\mathrm{SC}$ & $\mathrm{SC}$ \\
\hline 6 - Altura significativa de onda & $\mathrm{SC}$ & $\mathrm{SC}$ & $\mathrm{SC}$ \\
\hline 7 - Amplitude de maré média & $\mathrm{SC}$ & $\mathrm{SC}$ & $\mathrm{SC}$ \\
\hline
\end{tabular}

*SC = Sem Classificação. Os valores das variáveis não apresentaram variação relativa ao longo dos setores da zona de praia. 
Na geração do mapa de vulnerabilidade (Figura 3) os valores de CVI calculados ocorreram entre 1,00 e 3,00 , com média 1,72 e desvio padrão 1,11 . Os $25^{\circ}, 50^{\circ}$, e $75^{\circ}$ percentis do CVI foram $1,08,1,15$, e 2,08, respectivamente. Assim, valores de CVI abaixo de 1,08 foram atribuídos à categoria de baixa vulnerabilidade, entre 1,08 e 2,08 de média vulnerabilidade, e maiores do que 2,08 de alta vulnerabilidade. Em termos quantitativos, dos 3,054 km de linha de costa, 25,6\% foi classificada como alta vulnerabilidade, $25,1 \%$ como média vulnerabilidade, e 49,3\% como baixa vulnerabilidade. Vale ressaltar que as 3 classes criadas a partir dos percentis do CVI representam as variações de vulnerabilidade (baixa, média, alta) a nível local, ou seja, não podem ser comparadas diretamente com os mesmos níveis de vulnerabilidades de outras regiões; para isso, seria necessário o cálculo do CVI com as variáveis determinadas e obtidas nas regiões distintas, desde que levantados com a mesma metodologia aplicada no presente trabalho.

Os resultados mostraram que as variáveis geológicas quantitativas foram as responsáveis pela variação relativa dos índices de vulnerabilidade, pois as demais variáveis (geológica qualitativa e de processos físicos) foram consideradas constantes ao longo de toda a costa. O setor S1 apresentou a combinação mais crítica das variáveis devido às menores declividades e elevações da praia e à maior taxa de erosão, indicando ser o setor mais susceptível à futura elevação do nível do mar, mostrando baixa resiliência e pouca capacidade de recuperação natural dos sedimentos erodidos. Assim, o método aplicado permitiu o realce de áreas onde o efeito conjunto de algumas variáveis influencia diretamente na vulnerabilidade à erosão costeira. Isso mostrou a importância da combinação dessas variáveis nas análises de vulnerabilidades que, quando analisadas isoladamente, podem não ser suficientes para representar adequadamente a complexa vulnerabilidade costeira.

Em termos metodológicos, o realce de setores com diferentes níveis de vulnerabilidade em área de pequenas dimensões só foi possível devido à adoção de metodologia de alta precisão na determinação das variáveis geológicas quantificáveis. Os perfis de praia georreferenciados e nivelados com precisão centimétrica em relação ao SGB permitiu os seguintes fatos: (i) que fossem levantados sempre nos mesmos locais, o que viabilizou a comparação multitemporal; e (ii) que tivessem precisão relativa centimétrica nas diferenças de nível ao longo de cada perfil de praia e entre os três perfis, adequada para a mensuração das diferenças de nível obtidas em campo, de ordem métrica. A Figura 4 ilustra perfis de praia georreferenciados e nivelados ao NMM, levantados em duas épocas diferentes do ano (verão e inverno) nos setores S1, S2 e S3; as diferenças de nível nos pontos iníciais dos perfis variaram entre $0,227 \mathrm{~m}$ (entre Perfil 01 e Perfil 02) e 0,676 m (entre Perfil 01 e Perfil 03).

Como indica a Figura 4 os perfis de praia são curtos (apoximadamente $40 \mathrm{~m}$ ), devido à substituição do pós-praia por obras de urbanização, com o nível da água atingindo o muro da orla na maré em preamar (com visualizado na Figura 2). Nesse caso, os pontos iniciais dos perfis de praia coincidem com o muro da orla. A falta de feições naturais de pós-praia responsáveis pela proteção costeira, como bermas e dunas frontais, fez com que a variável geomorfológica fosse crítica em termos de vulnerabilidade em todos os setores

No Perfil 01, de menor altitude em relação aos demais (e maior vulnerabilidade), a partir da distância de $20 \mathrm{~m}$ praticamente não ocorre variação da altitude ortométrica com a distância, evideciando a intensa erosão costeira nesse trecho. 


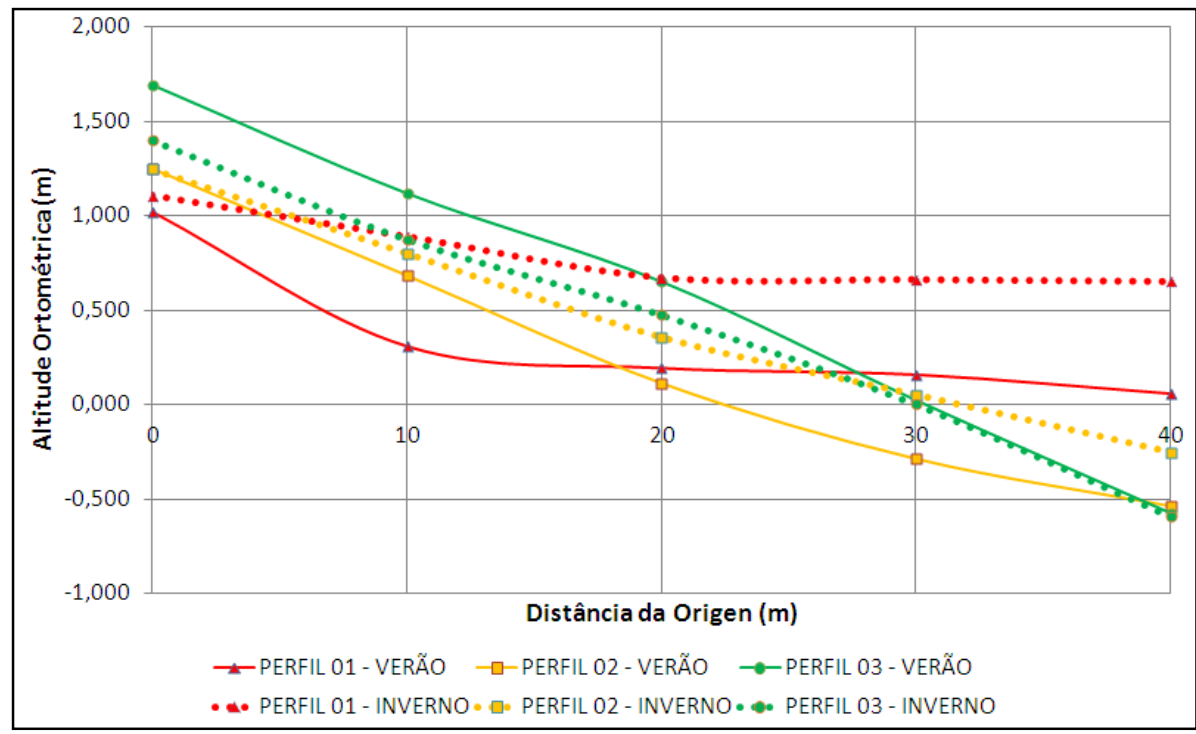

Figura 4: Perfis de praia levantados em Fevereiro (verão) e Julho (inverno) de 2013, nos setores S1 (Perfil 01), S2 (Perfis 02) e S3 (Perfil3), em que se nota o padrão de variação sazonal da morfologia da área de estudo.

\subsection{Impactos decorrentes da elevação do nível do mar}

A vulnerabilidade costeira à elevação do NMM expressou a necessidade de maior ou menor proteção do meio físico em relação às alterações provocadas pela aceleração do processo erosivo, que, no estudo de caso, foi influenciado principalmente pelas variáveis geológicas quantitativas. No entanto, segundo o Ministério do Meio Ambiente (MMA, 2008) e Egler (1996) a existência de um risco, associado a um evento que pode se realizar ou não, só se constitui quando há valoração de algum bem, material ou imaterial, pois não há risco sem a estimativa de perda. Como a elevação do nível médio do mar é um fato esperado para o decorrer das décadas futuras, não se prevê que ocorram perdas humanas com a aceleração do processo erosivo, pois a sociedade terá tempo suficiente para adaptação a esses desastres. No entanto, se não forem aplicadas intervenções locais para a contenção da erosão e preservação costeira, poderão ocorrer danos socioeconômicos e ambientais.

Assim, na implantação de intervenções de prevenção e/ou mitigação de impactos socioeconômicos e ambientais da faixa litorânea, a gestão pública deve analisar a relação espacial entre a vulnerabilidade e os danos. Por exemplo, os riscos associados às atividades industriais localizadas nas proximidades da zona de praia dos setores Oeste e Leste devem ser estimados de formas peculiares quanto ao tipo de intervenção, pois possuem níveis diferentes de vulnerabilidades - o setor Oeste tem prioridade em caso de intervenções por possuir maior vulnerabilidade quanto a elevação do nível do mar. Por outro lado, em áreas distintas com igual nível de vulnerabilidade e diferentes usos do solo deve-se avaliar o dano provocado pela aceleração do processo erosivo. Nesse caso, no setor Leste, onde a vulnerabilidade é baixa, espera-se que o risco associado à atividade industrial seja diferente em relação à atividade turística, pois o dano socioeconômico e ambiental provocado pela remoção das instalações antrópicas é função das características de cada uso do solo. 
Dessa forma, a partir das análises integradas de vulnerabilidade e dano, os órgãos responsáveis pela gestão costeira podem reduzir o risco da população de duas formas: i) redução do potencial de dano em áreas de alta vulnerabilidade natural, pela alteração do uso e ocupação do solo com a remoção de instalações (residenciais, industriais, etc.); ii) redução da vulnerabilidade natural em áreas com alto potencial de dano, caso não seja viável ou possível a alteração do uso e ocupação do solo; nesse caso, aplicando-se, por exemplo, intervenções para contenção de erosão como obras de engenharia e/ou processos de adição de sedimentos e vegetação ao sistema praial. No entanto, seja qual for a estratégia adotada para a redução de vulnerabilidade ou potencial de dano, estudos minuciosos, com obtenção de séries temporais contínuas e longas sobre os padrões e regimes de dinâmica costeira, devem ser realizados para que a tomada de decisão seja realizada da maneira fundamentada.

Apesar dos impactos previstos em decorrência da elevação do nível do mar, deve-se destacar o papel das instalações antrópicas na própria aceleração do processo erosivo ao longo do tempo. Os conjuntos residenciais, as estruturas turísticas e as instalações industriais foram construídas em setores de pós-praia, uma zona de armazenamento e aporte sazonal de sedimentos para a faixa de praia, ou seja, de acúmulo de células sedimentares. A ocupação de tais trechos impede a ação completa e efetiva dos processos costeiros sobre a redistribuição dos sedimentos costeiros, afetando o sistema de defesa natural da zona de praia contra a ação erosiva - a interrupção da dinâmica natural dos sedimentos deslocando-se no sistema praia-duna acarreta intensificação da ação erosiva e altera a dinâmica natural dos ciclos ambientais. $\mathrm{O}$ aumento na ocupação da orla marítima e a implantação de atividades industriais em áreas de células sedimentares importantes geraram a baixa resiliência no ambiente praial da Ilha de Madre de Deus.

\section{CONCLUSÕES}

A metodologia empregada no presente trabalho permitiu a delimitação de setores da orla costeira com diferentes níveis de vulnerabilidade à elevação do NMM, em escala local, com realce dos locais prioritários a intervenções de prevenção e/ou mitigação de impactos. Esse nível de detalhamento do mapeamento em área de dimensões relativamente pequenas tornou a metodologia adequada para a gestão costeira de municípios de pequeno porte e/ou com recursos financeiros limitados.

A delimitação desses setores em área de pequenas dimensões só foi possível devido à integração de dados topográficos e geodésicos de alta precisão na determinação dos perfis de praia, que foram georreferenciados e nivelados com precisão centimétrica em relação ao SGB. Essas características permitiram precisão relativa centimétrica nas diferenças de nível, tanto ao longo de cada perfil quanto entre os perfis distribuídos ao longo da orla costeira, adequada para a mensuração das diferenças de nível obtidas em campo, de ordem métrica.

Com a efetividade da delimitação dos diferentes níveis de vulnerabilidade, ficou clara a necessidade da estimativa dos efeitos da elevação do nível do mar nas atividades antrópicas instaladas na orla marítima, com mensuração de impactos devido à adaptação e/ou mitigação de tais atividades. Para essa finalidade os modelos estáticos de elevação do NMM podem ser de grande utilidade, pois permitem identificar áreas de abrangência dessa elevação para diferentes cenários futuros em função da topografia da zona de praia adjacente e de prognósticos de elevação do nível do mar. 
A partir do mapa de vulnerabilidade costeira à elevação do NMM as autoridades devem repensar a gestão costeira da orla marítima da Ilha de Madre de Deus para redução dos riscos potenciais decorrentes da elevação futura. Duas possiblidades são factíveis: redução da vulnerabilidade da zona de praia com a aplicação de intervenções antrópicas de contenção de erosão, como estruturas de engenharia ou adição de sedimentos e vegetação ao sistema; ou remoção de instalações antrópicas localizadas nas proximidades da zona de praia. Seja qual for a estratégia, estudo minucioso deve ser realizado para o entendimento da dinâmica praial e de como as instalações antrópicas se relacionam com o meio ambiente, para que as tomadas de decisão sejam realizadas de maneira fundamentada.

\section{AGRADECIMENTOS}

Os autores agradecem ao Conselho Nacional de Desenvolvimento Científico e Tecnológico (CNPq) pela concessão de bolsas de estudo do Programa Institucional de Bolsas de Iniciação Tecnológica (PIBITI).

\section{REFERÊNCIAS BIBLIOGRÁFICAS}

Amaro, Venerando E., Santos, Marcelo S.T., e Souto, Michael V.S. Geotecnologias aplicadas ao monitoramento costeiro: Sensoriamento remoto e Geodésia de precisão. Natal: Editora Natal, 2012.

Barboza, Andressa A. "Análise da dinâmica praial e estratégias de prevenção ou mitigação de danos à zona de praia de Madre de Deus/BA". Trabalho de Conclusão de Curso, Universidade Federal do Recôncavo da Bahia, 2014.

Boori, Mukeshi S., e Amaro, Venerando E. "Natural and eco-environmental vulnerability assessment through multi-temporal satellite data sets in Apodi valley region, Northeast Brazil". Journal of Geography and Regional Planning 4 (2011a): 216-230. Acesso 01/11/2014. http://www.academicjournals.org/journal/JGRP/edition/April_2011.

Boori, Mukeshi S., e Amaro, Venerando E. "A remote sensing approach for vulnerability and environmental change in Apodi valley region, Northeast Brazil". International Journal of Environmental, Earth Science and Engineering 5 (2011b): 01-11. Acesso 01/11/2014. http://www.waset.org/publications/7306.

Boruff, Bryan J., Emrich, Christopher, e Cutter, Susan L. "Erosion hazard vulnerability of US coastal counties". Journal of Coastal Research 21 (2005): 932-942. Acesso 01/11/2014. doi:10.2112/04-0172.1.

Bradley, Sean P., Dantas, Z.M., e Saldanha, M.G. "Gestão ambiental de uma Cidade Indústria: O caso de Madre de Deus. Salvador/BA". Trabalho de Conclusão de Curso (Especialização em Gerenciamento e Tecnologias Ambientais na Indústria), Universidade Federal da Bahia, 2010.

DHN (DIRETORIA DE HIDROGRAFIA E NAVEGAÇÃO). "Carta náutica 1105: Porto de Madre de Deus". Accesso 10/01/2013. https://www.mar.mil.br/dhn/chm/cartas/download/cartasbsb/cartas_eletronicas_Internet.htm. 
Egler, Cláudio A. G. "Risco Ambiental como critério de gestão do território. Uma aplicação à zona costeira Brasileira". Território 1 (1996): 31-41. Acesso 01/11/2014. http://www.laget.eco.br/index.php?option=com_wrapper\&view=wrapper\&Itemid=6.

Espartel, Lelis. Caderneta de Campo. Porto Alegre: Editora Globo, 1983.

Featherstone, Will E., Dentith, Michael C., e Kirby, Jon F. "Strategies for the accurated determination of orthometric heights from GPS". Survey Review 34 (1998): 278-296. Acesso 01/11/2014. http://www.surveyreview.org/index.html

Freire, Jamile T., Santos, R.W.N., e Santos, A.S. "Madre de Deus, o risco e a gestão em uma cidade indústria". Trabalho apresentado no Seminário Urbanismo na Bahia: A produção da Cidade e a Captura do Público: que perspectivas?, Bahia, Salvador, 07-09 novembro, 2012.

Gornitz, V.M.; Daniels, R.C.; White, T.W.; Birdwell, K.R. "The development of a coastal risk assessment database: Vulnerability to sea-level rise in the U.S. Southeast". Journal of Coastal $\begin{array}{lllll}\text { Research } & 12 & \text { (1994): 327-338. Acesso }\end{array}$ http://www.jcronline.org/page/special_publications

Gorokhovich, Yuri, Leiserowitz, Anthony, e Dugan, Darcy. "Integrating Coastal Vulnerability and Community-Based subsistence resource mapping in Northwest Alaska". Journal of Coastal Research 30 (2014): 158-169. Accesso 01/11/2014. doi: 10.2112/JCOASTRES-D-13-00001.1.

Hammar-Klose E.S., Pendleton, E.A., Thieler, E.R, e Williams, SJ. Coastal Vulnerability Assessment of Cape Cod National Seashore (CACO) to Sea-Level Rise. Reston: U.S. Geological Survey, Open file Report 02-233, 2003.

Hatje, Vanessa, e Andrade, Jaílson B. Baía de Todos os Santos: Aspectos oceanográficos. Salvador: Editora da UFBA - EDUFBA, 2009.

IBGE (FUNDAÇÃO INSTITUTO BRASILEIRO DE GEOGRAFIA E ESTATÍSTICA). "Informações obtidas da página do IBGE na internet". Acesso 01/03/2013. http://www.ibge.gov.br

IPCC, 2013. Climate Change 2013: The Physical Science Basis. Contribution of Working Group I to the Fifth Assessment Report of the Intergovernmental Panel on Climate Change, Stockholm, 2013.

Kobiyama, Mosato, Mendonça, Magaly, Moreno, Davis A., Marcelino, Isabela P. V. O., Marcelino, Emerson V., Gonçalvez, Edson F., Brazetti, Leticia L. P., Goerl, Roberto F., Molleri, Gustavo S. F., e Rudorff, Frederico M. Prevenção de Desastres Naturais: Conceitos Básicos. Curitiba: Editora Organic Trading, 2006.

Kumar, T. Srinivasa, Mahendra, R.S.; Nayak, Shailesh, Radhakrishnan, K., e Sahu, K.C. Coastal vulnerability assessment for Orissa State, east coast of India. Journal of Coastal Research 26 (2010): 523-534, 2010. Acesso 01/11/2014. doi: 10.2112/09-1186.1

Loch, Carlos, e Cordini, Jucilei. Topografia Contemporânea. Florianópolis: Editora UFSC, 2007.

MMA (Ministério do Meio Ambiente). Macrodiagnóstico da Zona Costeira e Marinha do Brasil. Brasília: MMA, 2008.

Monico, João F.G. Posicionamento pelo GNSS: descrição, fundamentos e aplicações. São Paulo: Editora Unesp, 2007.

Pendleton, E.A., Barras, J.A., Williams, S.J., e Twichell, D.C. Coastal Vulnerability Assessment of the Northern Gulf of Mexico to Sea-Level Rise and Coastal Change. Reston: U.S. Geological Survey, Report Series 1146, 2010. 
Santos, Marcelo S.T., e Amaro, Venerando E. "Dinâmica sazonal de processos costeiros e estuarinos em sistema de praias arenosas e ilhas barreira no Nordeste do Brasil". Revista Brasileira de Geomorfologia 14 (2013): 151-162. Acesso 01/11/2014. http://www.lsie.unb.br/rbg/index.php/rbg/article/download/298/325.

Santos, Marcelo S.T., Amaro, Venerando E., Ferreira, Anderson T.S., e Santos, André L.S. "Altimetria GNSS de precisão aplicada ao monitoramento da dinâmica sedimentar costeira de curta duração em escala regional”. Boletim de Ciências Geodésicas 19 (2013): 624-638. Acesso 01/11/2014. http://dx.doi.org/10.1590/S1982-21702013000400007.

Santos, Marcelo S.T., e Amaro, Venerando E. "Rede geodésica para o monitoramento costeiro do Litoral Setentrional do Estado do Rio Grande do Norte". Boletim de Ciências Geodésicas 17 (2011): 571-585. Acesso $01 / 11 / 2014$ http://ojs.c3sl.ufpr.br/ojs2/index.php/bcg/article/view/25401/17021

Santos, Marcelo S.T., Amaro, Venerando E., e Souto, Michael V. S. "Metodologia geodésica para levantamento de linha de costa e modelagem digital de elevação de praias arenosas em estudos de precisão de geomorfologia e dinâmica costeira". Revista Brasileira de Cartografia 63 (2011): 469-483. Acesso 01/11/2014. http://www.lsie.unb.br/rbc/index.php/rbc.

Seeber, Gunter. Satellite Geodesy: Fundations, methods and applications. N. York: Walter de Gruyter, 1993.

Shaw, J., Taylor, R.B., Forbes, D.L., Ruz, M.H., e Solomon, S. Sensitivity of the Canadian Coast to Sea-Level Rise. Alberta: Geological Survey of Canada Bulletin 505, 1998.

Shidawara, Masatoshi. Flood hazard map distribution. Urban Water 1 (1999): 125-129. Acesso 01/11/2014. http://www.sciencedirect.com/science/article/pii/S1462075800000029.

Thieler, E. Robert., e Hammar-Klose, Erika S. National Assessment of Coastal Vulnerability to Sea-Level Rise: U.S. Atlantic Coast. U.S. Geological Survey. Reston: U.S. Geological Survey, Open file Report 99-233, 1999.

Recebido em dezembro de 2014.

Aceito em junho de 2015. 